\title{
ESTRATEGIAS QUE PROMUEVEN ESTILOS DE VIDA SALUDABLES
}

\section{STRATEGIES THAT PROMOTE HEALTHY LIFESTYLES}

\author{
Rosa del Carmen Coral ${ }^{1}$, Luz Dalila Vargas ${ }^{2}$ \\ ${ }^{1}$ Enfermera. Magíster en Docencia Universitaria. Docente Facultad de Enfermería U.D.C.A, calle 222 No. 55-37, Bogotá, \\ D.C., Colombia, e-mail: rcoral@udca.edu.co; ${ }^{2}$ Enfermera. Magíster en Enfermería con Énfasis en Gerencia de Servicios de \\ Salud. Docente Facultad de Enfermería U.D.C.A, calle 222 No. 55-37, Bogotá, D.C., Colombia, e-mail: luvargas@udca.edu. \\ co
}

Rev. U.D.C.A Act. \& Div. Cient. 17(1): 35-43, Enero-Junio, 2014

\section{RESUMEN}

La formación de futuros profesionales de enfermería debe incluir el fortalecimiento de estilos de vida saludables (EVS), para aplicarlos en el diario vivir y en su desempeño. Se realizó una investigación en dos fases: en la primera, se identificaron problemáticas y estrategias en relación a estilos de vida saludables y, en la segunda, se implementaron y se evaluaron estrategias, para fomentar estilos de vida saludables en estudiantes de enfermería. Este artículo muestra los resultados de la segunda fase. El diseño corresponde a Investigación Acción Participación (IAP). El grupo de estudio fue conformado por 237 estudiantes. El muestreo fue intencional, de acuerdo a criterios de inclusión. La recolección de la información, se realizó con entrevistas a grupos focales, diarios de campo, videos, entrevistas a profundidad y encuesta semi-estructurada. Para el análisis de datos, se utilizó el Software ATLAS.Ti (Qualitative Data Analysis) y el software IBM SPSS Statistics 19. Los resultados muestran que los estudiantes conocen los estilos de vida saludables; sin embargo, falta ponerlos en práctica; se presentan cambios en los estilos relacionados con el abandono del consumo de cigarrillo y prácticas saludables. Se concluye que, para lograr cambios significativos y sostenibles en el tiempo, se requiere implementar estrategias que permitan espacios de reflexión y de sensibilización; de igual manera, exige el ejemplo, el acompañamiento y el compromiso permanente por parte de los docentes, de los estudiantes como pares, así como también de la Universidad. Estudiar enfermería favorece los estilos de vida saludables de los estudiantes.

Palabras clave: Estudiantes, promoción de la salud, conducta alimentaria, hábitos, conductas saludables.

\section{SUMMARY}

The training of future nursing professionals should include the strengthening of healthy lifestyles to apply to daily life and performance as professionals. A research was conducted in two phases: the first to identify issues and strategies in relation to healthy lifestyles, the second, implementing and evaluating strategies to promote healthy lifestyles among nursing students. This article shows the results of the second phase of the investigation. The design corresponds to Participation Action Research (PAR). The study group was comprised of 237 students. Sampling was intentional according to inclusion criteria. The data collection was performed with focus group interviews, field notes, videos, interviews and indepth semi-structured questionnaire. For data analysis the Software ATLAS. Ti (Qualitative Data Analysis) and IBM SPSS Statistics 19 software was used. The results show that students know the healthy lifestyles, changes occur in the styles related to the cessation of smoking and health practices. It is concluded that to achieve meaningful and sustainable changes in time, strategies that allow reflection and awareness are required to be implemented, also examples are needed, as well as permanent support and commitment from teachers, students and peers and by the University. Studying nursing encourages healthy lifestyles of students.

Key words: Students, health promotion, feeding behavior, habits, healthy behavior.

\section{INTRODUCCIÓN}

La docencia en enfermería, a través de un enfoque crítico constructivista, se dirige al desarrollo cognitivo- racional, emocional y volitivo de los estudiantes, para mejorar su forma de ser y de vivir hacia la autonomía, es decir, involucra 
dentro de la formación integral: el desarrollo humano, la calidad de vida, los factores individuales, sociales, políticos, económicos y culturales, que determinan el estilo de vida de los sujetos y, por consiguiente, su implicación en el estado de la salud (Palencia, 2006).

El concepto de estilo de vida, según Reyes et al. (2011), se relaciona con modo de vida, determina la forma cómo se desarrolla el hombre dentro de la sociedad y su relación con el proceso salud - enfermedad. Son patrones de comportamiento de las personas que presentan una relativa permanencia en el tiempo, influenciado por factores internos: pensamientos, emociones, conocimientos, habilidad y actitudes y externos: condiciones familiares, sociales, culturas y medioambientales (Escobar \& Pico, 2013). Manifiesta una determinada cultura en una comunidad, familia o individuo, que tiene una forma de vida en particular, de acuerdo a sus conocimientos, sus necesidades y sus posibilidades económicas (Vives, 2007).

Los estilos de vida saludables están relacionados con la disminución de la probabilidad de adquirir enfermedades crónicas no transmisibles y de mejorar la calidad de vida; se adquieren en la infancia, pero también en la vida universitaria. Los jóvenes, en sus años universitarios, son sensibles a la última moda y a buscar nueva ideas; la formación en hábitos de vida saludables es base para la salud y el bienestar, así como para una esperanza de vida saludable (Jeongsoo, 2014).

Según Espinoza et al. (2011), el inicio de la vida universitaria, es una etapa marcada por una serie de cambios; al respecto, González et al. (2014) consideran que el estrés y las cargas de horario conducen a comidas rápidas y poco nutritivas; la irregularidad en los horarios de alimentación y la falta de tiempo para realizar ejercicio inciden en los hábitos de vida, los higiénico-dietéticos y la actividad física.

Los resultados de investigaciones sobre los estilos de vida de estudiantes universitarios, afirman que tan sólo 13,6\% tienen alimentación adecuada, 28,7\% asumen factores de riesgo por trastornos de la conducta alimentaria, $24,7 \%$ presentan impulsividad, $40 \%$ muestran problemas de ansiedad, 7,7\% evidencian depresión, 14,8\% tienen riesgo suicida, 62,9\% disfunción familiar entre leve y severa (Páez Cala \& Castaño Castrillón, 2010) y 42,7\% de los hombres y 29,8\% de las mujeres realizan ejercicio en programas de actividad física en la universidad (Espinoza et al. 2011).

Otro estudio realizado en Inglaterra muestra que los estudiantes tienen estilos de vida pocos saludables: el $70 \%$ informaron que no realizan ejercicio cuatro veces a la semana; el $56 \%$ informaron consumo excesivo de alcohol, al menos una vez a la semana; $66 \%$ no comer la cantidad recomendada de, al menos, cinco porciones de frutas y de verduras por día y $24 \%$ manifestaron consumo de cigarrillo; también resalta este estudio, la importancia de la relación entre el estrés psicológico y las conductas no saludables (Dodd et al. 2010).

En el caso de estudiantes del área de la salud, los estudios coinciden en que la práctica regular de ejercicio suele ser una de las debilidades encontradas en los estilos de vida de estas poblaciones, a pesar del conocimiento sobre su relevancia frente a la prevención de las enfermedades cardiovasculares, ansiedad, depresión y sus beneficios en la salud mental y en la calidad de vida (Arguello et al. 2009).

Para las estudiantes de enfermería, la inactividad física y los desórdenes alimenticios tienen una importante prevalencia (Cancel \& Ayán, 2011). La actividad física y la dieta están relacionados; sin embargo, la actividad es la variable más importante para la salud; las mujeres estudiantes universitarias, al igual que las adultas, tienen los mismos factores de riesgo para la salud, debido a un estilo de vida inactivo (Jeongsoo, 2014). Un meta-análisis encontró que las intervenciones para reducir el comportamiento y las conductas sedentarias da lugar a una disminución significativa de éstas (Grieken et al. 2012).

Otra problemática en estudiantes de enfermería es el consumo de cigarrillo y drogas. Al respecto, Zárate et al. (2006) evidencian que el $71,8 \%$ había consumido alguna vez en la vida; este dato es mayor a lo reportado para la población general urbana en Perú, pero menor comparado con otras facultades y otros países; estudios internacionales reportan que en estudiantes de enfermería y de medicina, las mujeres son las que más fuman.

En relación a esta problemática, se presentan los resultados del trabajo de investigación que permitió fortalecer estrategias para la toma de decisiones en estilos de vida saludables, con un grupo de estudiantes de la Facultad de Enfermería. Se analizaron las prácticas de estilos de vida saludables que realizan; se definieron, implementaron y evaluaron estrategias para fortalecerlas.

\section{MATERIALES Y MÉTODOS}

La investigación, se desarrolló en el paradigma cualitativo, diseño de Investigación Acción Participación (IAP). Este tipo de estudio, se caracteriza porque permite la construcción del conocimiento y el compromiso en la solución de problemáticas sociales que involucran a los afectados (Carrillo, 1998); al respecto, Leal (2009) afirma: "el conocimiento da poder a estos actores, en la medida que aumenta su conciencia; es decir, su conocimiento". 
La población corresponde a 438 estudiantes de la Facultad de Enfermería, de los cuales, se tomó una muestra de 237 $(54,11 \%)$. El muestreo fue no probabilístico e intencional, de acuerdo al criterio de inclusión: estudiantes que participaron voluntariamente en la investigación en una o más de las estrategias implementadas.

El proceso de investigación, de acuerdo al diseño IAP, incluyó tres momentos: identificación con el grupo de estudio de los estilos de vida saludables, propuesta e implementación de estrategias y evaluación de las estrategias implementadas.

Las técnicas e instrumentos para la recolección de la información fueron: Entrevista a grupo focal y diario de campo, aplicados a 29 estudiantes (12,24\%); entrevista a profundidad, practicada a 13 estudiantes $(5,49 \%)$, quienes participaron en la estrategia de proyecto de vida; encuesta semiestructurada, aplicada a (237) estudiantes, para evaluar los cambios que se presentaron con la implementación de estrategias en los estilos de vida saludables.

Las estrategias implementadas fueron: Proyecto de Vida; talleres sobre estilos de vida saludables: autocuidado, prevención y abandono del consumo de drogas, actividad física, nutrición, sueño y descanso y espiritualidad; conferencias brindadas por docentes investigadores; conferencia brindada por nutricionista; jornadas de medición: peso, talla, IMC y clasificación de riesgo cardiovascular; asesoría personalizada por docentes investigadores a estudiantes y remisión a médico, psicólogo o nutricionista y, finalmente, jornadas mensuales de actividad física, denominada: "Hora Loca por la Salud".

La sistematización y análisis de la información, se realizó con el Software ATLAS. Ti: (Qualitative Data Analysis) y el Software IBM SPSS Statistics 19.

Consideraciones éticas: Se solicitó el consentimiento informado al grupo de estudio. El proyecto fue aprobado por el Comité de la Facultad de Enfermería y de la Universidad. Los estudiantes participaron libremente y firmaron el consentimiento informado. El estudio, se clasificó en riesgo mínimo, porque no se realizaron intervenciones que afectaran la integridad física, social o emocional de los participantes. Se tuvo en cuenta la Resolución 8340 del Ministerio de Salud y los principios de la Declaración de Helsinki y la CIOMS para la investigación con personas.

\section{RESULTADOS Y DISCUSIÓN}

En los resultados de la aplicación de la entrevista al grupo focal, los estudiantes expresan con mayor frecuencia las siguientes prácticas, como estilos de vida saludables: Ade- cuada nutrición (28), actividad física (23); descanso (13), no consumo de alcohol o cigarrillo (10), equilibrio emocional (6), control médico (5) higiene personal (4); evitar estrés (2), buenas relaciones interpersonales (2), recreación (2), ergonomía (1), técnicas de relajación (1), estudiar y leer (1).

Si bien es cierto, que conocen la importancia de los estilos de vida saludables, muchos de ellos no los practican (10), otros refieren que saben lo que tienen que hacer (6), consideran que la profesión facilita la adquisición de hábitos saludables (9), mencionan el compromiso con la profesión en la promoción de los estilos de vida para mejorar la calidad de vida de las personas (3). Algunos testimonios refieren:

\section{"Estamos estudiando, sabemos lo que deberíamos hacer y no lo hacemos". \\ "Nosotros como personas que promovemos la salud deberíamos cuidarnos más". \\ "No fumo, porque creo que si uno le dice a alguien que no haga eso, es porque uno no lo está haciendo, eso es ético".}

En relación a los hábitos nutricionales, refieren que tienen conocimiento, pero hay dificultades para mantener una adecuada nutrición por la escasez de dinero, falta de tiempo, poco acceso a alimentos saludables en la Universidad, falta de voluntad y también refieren que influye el estado de ánimo. Comentan:

\section{"Estamos expuestas a la comida chatarra". "Como comida chatarra, pero compenso comiendo comida saludable".}

Estos resultado coinciden con los registrados por Rizo et al. (2014), quienes afirman que los estudiantes no modifican sus hábitos alimentarios a pesar de tener la información suficiente para hacerlo; existe desequilibrio en la dieta y el consumo de micronutrientes. Espinoza et al. (2011) mencionan otras situaciones relacionadas con la preferencias que tienen los estudiantes; encontraron que las mujeres compran y consumen, como primera elección, golosinas, a diferencia de los hombres, que prefieren los lácteos, las bebidas y los productos con fibra.

En relación a la actividad física, el grupo de estudio expresó diferentes dificultades para ponerla en práctica: falta de tiempo (8), carga académica (5), distancias y transporte (2), compromisos familiares (1) cansancio (1) o simplemente no les gusta realizar actividad física (1). Refieren:

"La carga académica no permite hacer ejercicio". "Depende de las distancias, además del desgaste del transmilenio, estrés en el transporte, es agotador". 
"Llego cansada y me acuesto, Influye el estado de ánimo, el tiempo, el clima, lo académico".

Al respecto, Monteiro \& Mancussi (2006) registran que los estudiantes no realizan actividad física por falta de tiempo, $64,04 \%$; no les gusta, $22,47 \%$ y falta de dinero, $11,24 \%$; sin embargo, Mosquera et al. (2011) encuentran que los estudiantes desean invertir su tiempo libre en deporte, 31,5\%; logran hacerlo, $25,2 \%$ y no cuentan con espacios deportivos, entrenadores e instalaciones apropiadas. 6,3\%. Rodríguez et al. (2013) perciben otras razones, además de la falta de tiempo, como llegar cansados de la universidad, falta de costumbre y pereza.

Cabe resaltar que los estudiantes asumen conductas de riesgo que pueden afectar, a corto, mediano o largo plazo, su estado de salud. Al respecto, Farinola \& Bazán (2011) registran altos niveles de conductas sedentarias y bajos de actividad física en estudiantes, situación que los expone a desarrollar enfermedades cardiovasculares, obesidad y muerte prematura. Schnettler et al (2013) incluyen datos en América Latina sobre la prevalecía de sobrepeso y obesidad en esta población, entre 10 y $48 \%$ y, asociado a este problema, se reporta hipercolesterolemia y elevado nivel de triglicéridos.

Las intervenciones destinadas a prevenir sedentarismo excesivo pueden contribuir a la prevención del sobrepeso; disminución global significativa en el comportamiento sedentario, así como disminución significativa en el IMC (Grieken et al. 2012).

En relación al consumo de alcohol y cigarrillo, el grupo de estudio expresa contradicciones y formas de pensar que, posiblemente, los llevan a asumir conductas de riesgo. Refieren:

"Es contradictorio, uno sabe que no se debe hacer".

"No me parece malo, el exceso es malo".

"Para muchas personas fumar y tomar les hace sentirse bien, ¿̇i es bueno para ellos porque es malo?".

"Todas las personas conocemos los riesgos del cigarrillo, cada uno elige lo que tiene que hacer".

"Estoy evitando las bebidas alcohólicas".

En jóvenes, se reporta el consumo de cigarrillo y otro tipo de drogas, siendo el alcohol la sustancia más utilizada, seguido del tabaco, cannabis y estimulantes (Pedrosa et al. 2011). En un estudio con estudiantes de enfermería en Valencia España, se encontró que las drogas más empleadas fueron el tabaco, bebidas mezcladas con alcohol, cerveza, vino y hachís (García et al. 2013).

Según Tercedor et al. (2007), el hábito del consumo de cigarrillo aumenta el riesgo de mortalidad por cáncer, enfermedades cardiovasculares, cerebro vasculares y respirato- rias crónicas; por su parte, Iglesias et al. (2013), comentan que se ha demostrado que los hábitos de los fumadores son menos saludables que los no fumadores. De acuerdo a lo anterior, se requiere establecer procesos de sensibilización y estrategias de seguimiento en los escenarios académicos, para lograr cambios significativos.

Al respecto, Velásquez et al. (2006) afirman la necesidad de realizar intervenciones de acuerdo a los intereses y a las necesidades de los estudiantes y citan el Modelo de Proceso de Adopción de Precauciones (PAP): para lograr cambios, se requiere de procesos desde el cognitivo-conductual de un estilo de vida de riesgo, hasta una etapa de protección en el que se mantiene la acción. Haines et al. (2013) sugieren que las estrategias de promoción de la salud y de control del tabaco deben considerar con más cuidado las perspectivas de los jóvenes adultos que fuman y poner de relieve la necesidad de estrategias que sean creíbles, para esta población en particular. Dodd et al. (2010) manifiestan que el diseño de intervenciones dentro de esta población, debe considerar el estrés psicológico, ya que esto desempeña un papel significativo en la vida de un estudiante y puede ser un factor subyacente de la adopción de múltiples comportamientos no saludables; además, que los comportamientos de salud no se deben considerar en forma aislada unos de otros.

Los estudiantes que plantearon el proyecto de vida expresaron los siguientes aspectos a mejorar, en relación a sus ESV: relaciones interpersonales (21), nutrición (10), controlar emociones (9), disminuir el consumo de cigarrillo (5) y de alcohol (3) y compromiso con la profesión en mejorar la calidad de vida de las personas (Figura 1).

Al finalizar el estudio, como resultado de la aplicación de la entrevista a profundidad los estudiantes, reconocieron que estudiar enfermería les permitió reflexionar sobre sus vidas (3), mejoraron las relaciones interpersonales (2), la nutrición (6), el control de emociones (3) y el aspecto espiritual (3); disminución del consumo de cigarrillo (5), de alcohol (7) y de otras sustancias (1); sin embargo, dos estudiantes manifestaron que abandonaron el consumo de cigarrillo y alcohol, pero reincidieron.

El mismo grupo mencionó que abandonaron el consumo de drogas por: motivación personal (7), auto reflexión (8), influencia de la familia (5), pensar en el futuro (3), conocimientos adquiridos en la profesión (3), participación en proyectos de investigación, trabajar con los estudiantes desde primer semestre (2). Finalmente, proponen darle continuidad al proyecto de vida (Figura 2).

Cabe mencionar, la importancia de la utilización del proyecto de vida como estrategia para lograr metas y objetivos. Al respecto, Hernández (2006) expresa que ha sido considera- 


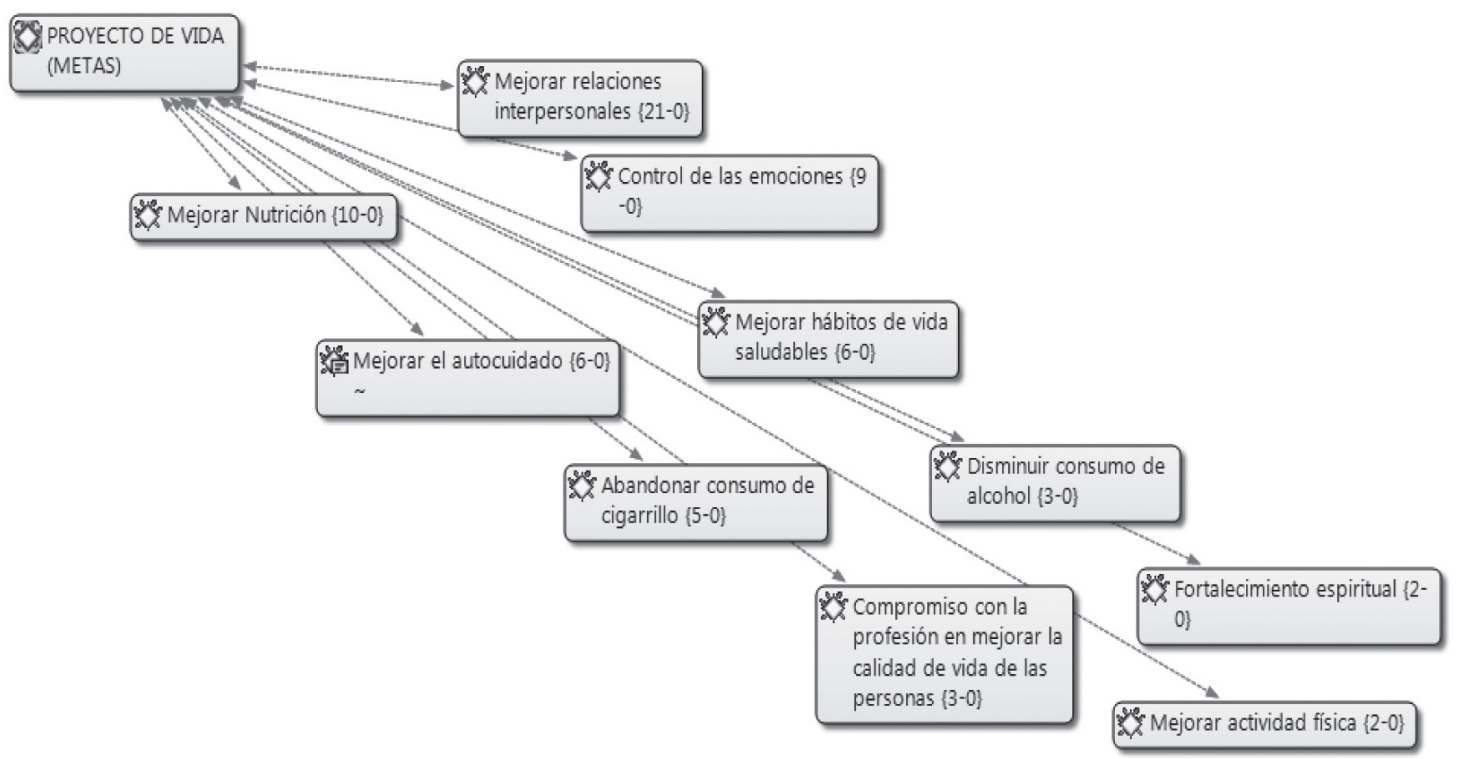

Figura 1. Resultados de frecuencia en expresiones sobre el Proyecto de Vida del grupo de estudio. Entre paréntesis frecuencia y códigos relacionados. Fuente análisis en Atlas Ti. 2012.

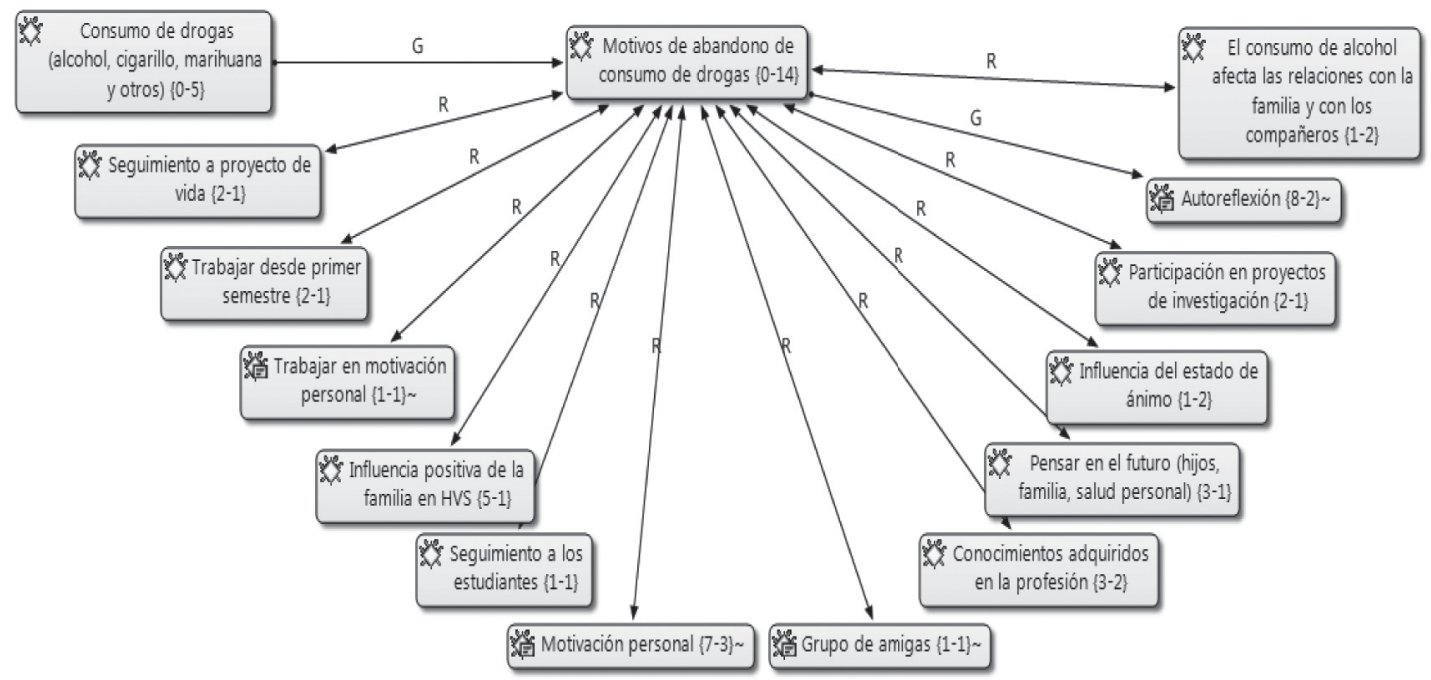

Figura 2. Motivos que tiene el grupo de estudio para abandonar las drogas. Fuente análisis en Atlas Ti. Entre paréntesis frecuencia y códigos relacionados. Entrevista a profundidad. 2012.

do como la "intención emancipatoria del desarrollo humano que expresa la apertura de la persona hacia el dominio del futuro, contribuye a delinear los rasgos de su estilo de vida personal y permite una representación clara de las metas y aspiraciones así como de las motivaciones que las inspiran".

En relación a los resultados de la aplicación de la encuesta semi-estructurada, la mayor participación en las estrategias implementadas corresponde a los estudiantes de los primeros semestres 175 (73,8\%); llama la atención, la menor participación de los estudiantes de semestres superiores, quienes deberían estar más comprometidos con su propio autocuidado (Gráfica 1). Al respecto, un estudio realizado por Jeongsoo, (2014) encontró que los estudiantes mayores tienen más desequilibrio por causa del trabajo académico, el estrés laboral y la inactividad en la vida diaria. 


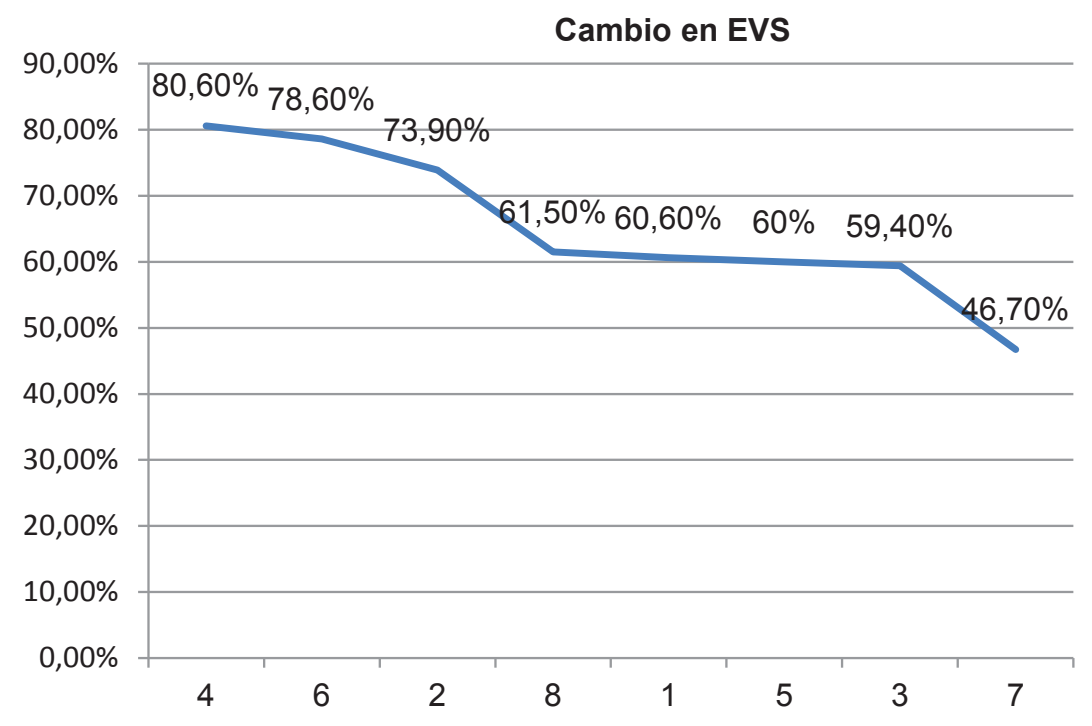

Gráfica 1. Porcentaje de estudiantes que manifestaron cambios positivos en Estilos de Vida saludables por semestre Fuente análisis en Excel de la Encuesta. U.D.C.A 2012.

Con relación a las estrategias que permitieron cambios en los estilos de vida saludables, 156 (66\%) estudiantes manifestaron realizar cambios. Las actividades en las que participaron los estudiantes, se observan en la gráfica 2, denota mayor inscripción en los controles de peso y talla realizados por los docentes investigadores, 60,3\% y en la jornada de actividad física "Hora Loca por la Salud", 56,7\%; al contrario ocurrió en las consultas con médico y nutricionista, 6,7\% y $13 \%$, respectivamente.

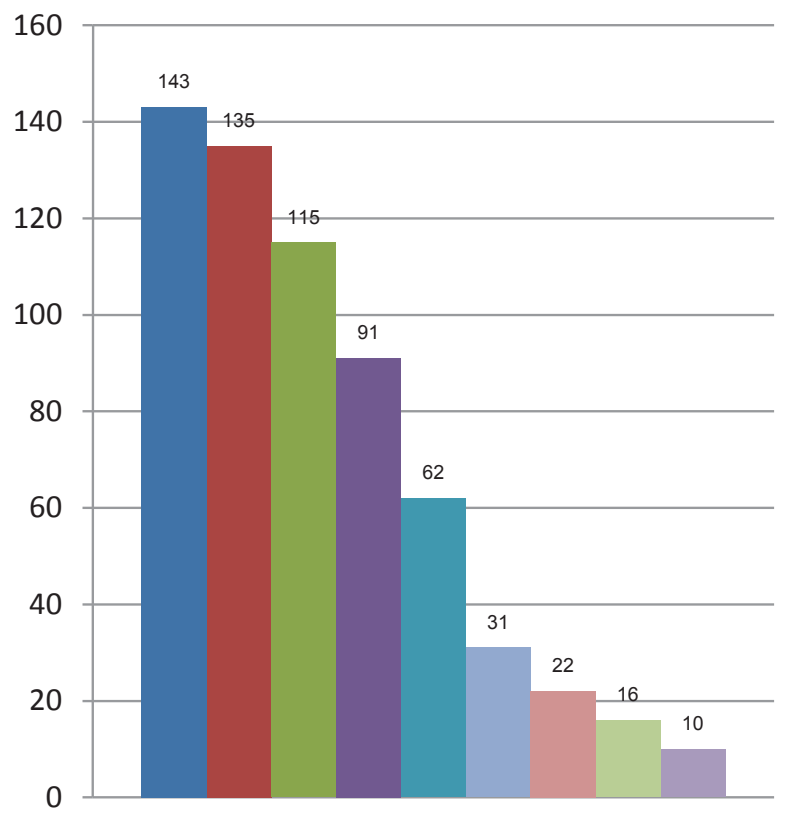

- Control de Peso y talla por investigadores

- Hora loca por la salud

- Talleres realizados por docentes investigadores

- Asesoria personalizada por docentes investigadores

- Conferencia por docentes investigadores

- Valoración por nutricionista

- Conferencia sobre Nutrición

- Control Médico

- Consulta Psicología

Gráfica 2. Participación de estudiantes de enfermería en estrategias para promover estilos de vida saludables (EVS). Fuente análisis en Excel de la Encuesta. 2012. 
Con relación a los estudiantes que participaron del control de peso y talla, $72,70 \%$ manifestaron cambios en sus EVS; $24,5 \%$ contestaron que no tuvieron cambios y $2,8 \%$, no respondieron. Por el contrario, el 51,7\% de los estudiantes que no participaron del control de peso y talla manifestaron cambios, frente a $46 \%$, que no manifestaron cambios.

Los estudiantes que asistieron a las sesiones de actividad física, 74,8\% manifestaron cambios en sus EVS; el 23,70\%, sin cambios y el $1,5 \%$, no respondieron. De 94 estudiantes $(39,6 \%)$ que no asistieron a la actividad física, el 51,10\%, manifestaron cambios. Se observa diferencia entre los estudiantes que asistieron a la hora loca y cambiaron sus EVS, 74,8\% y los que no asistieron, pero manifestaron cambios, $51,10 \%$.

Los estudiantes que participaron en los talleres sobre EVS reportaron cambios en sus EVS, en un $72,2 \%$; sin cambios, en el $25,2 \%$ y no respondieron, $2,60 \%$. Al contrario, los estudiantes que no participaron no tuvieron cambios, $44,4 \%$; tuvieron cambios, 56,9\% y no respondieron, 3 .

De los estudiantes que participaron de la asesoría personal por parte de las investigadoras, el 82,3\% manifestaron cambios en sus EVS, frente al $52,2 \%$ de estudiantes que no participaron, pero tuvieron cambios.

Los estudiantes que asistieron a las conferencias brindadas por las investigadoras manifestaron cambios en EVS, en un $74,2 \%$, frente a $60,7 \%$, que tuvieron cambios, pero que no participaron de las conferencias; en coherencia, el $21 \%$ que asistieron no tuvieron cambios, frente a un porcentaje más alto de $37,4 \%$, que no asistieron y no percibieron cambios.

En general, las actividades donde los estudiantes manifestaron que obtuvieron cambios en sus estilos de vida saludables fueron en el control de peso y talla, $43,9 \%$, seguido de la Hora Loca por la Salud, $42,6 \%$, talleres, $35 \%$ y asesoría personalizada, $31,6 \%$.

Los cambios más relevantes que los estudiantes han manifestado con la participación en las estrategias implementadas por el proyecto, se relacionan con la actividad física, $35 \%$; asistieron al gimnasio, 3,4\%; mejoraron la nutrición, $38 \%$; abandonaron el consumo de cigarrillo, $4,6 \%$; mejoraron el sueño y descanso, $5,4 \%$, entre otros.

En el presente estudio, se observó que los resultados en general fueron positivos; sin embargo, otros autores reportan lo contario, como el estudio realizado por Brandao et al. (2011), donde registraron que los jóvenes tenían riesgo de sobrepeso, obesidad, tabaquismo, sedentarismo, dislipidemia e hipertensión; el nivel de educación, según los autores, no parece tener un efecto protector sobre la adopción de es- tilos de vida saludables o estar inscritos en áreas de Ciencias de la Salud; de igual manera, el seguimiento a los estudiantes no mostró un efecto positivo.

Los resultados muestran que los estudiantes conocen los estilos de vida saludables; no obstante, falta ponerlos en práctica. Se presentan cambios en los estilos relacionados con el abandono del consumo de cigarrillo y prácticas saludables.

Para lograr cambios significativos y sostenibles en el tiempo, se requiere implementar estrategias que permitan espacios de reflexión y de sensibilización; de igual manera, exige el ejemplo, el acompañamiento y el compromiso permanente por parte de los docentes, de los estudiantes como pares, así como también de la Universidad y la inclusión en los planes de estudio de cursos académicos, que fomenten los estilos de vida saludables. Estudiar enfermería favorece el conocimiento y la adquisición de los estilos de vida saludables de los estudiantes. Se requiere el compromiso de docentes y de la universidad para fortalecerlos.

Agradecimientos: Las autoras agradecen a la Universidad, por el apoyo en la financiación del estudio; a los compañeros de la Facultad de Enfermería, que participaron en las estrategias implementadas; Bienestar Universitario, servicio médico y a la Facultad de Ciencias del Deporte. Conflicto de intereses: El manuscrito fue preparado y revisado con la participación de todos los autores, quienes declaramos que no existe ningún conflicto de intereses que ponga en riesgo la validez de los resultados presentados. Financiación: Este estudio fue financiado por la Universidad de Ciencias Aplicadas y Ambientales U.D.C.A.

\section{BIBLIOGRAFÍA}

1. ARGUELLO, M.; BAUTISTA, Y.; CARVAJAL, J.; DE CASTRO, K.; DÍAZ, D.; ESCOBAR, M.; GÓMEZ, L.; MORALES, M.; PINTO, C.; RINCÓN, L.; RUEDA, P.; SERRANO, J.; SUÁREZ, P.; TOLOZA, Z.; AGUDELO, D. 2009. Estilos de vida en estudiantes del área de la salud de Bucaramanga. Rev. de psicología de la Universidad de Antioquía. (Colombia). 1 (29):27-44. Disponible desde Internet en: http://aprendeenlinea. udea.edu.co/revistas/index.php/psicologia/article/ view/10019/9236 (con acceso 07/4/2014).

2. BRANDAO, M.; PIMENTEL, F.; CARDOSO, M. 2011. Impact of academic exposure on health status of university students. Rev. Saúde Pública. (Brasil). 45(1):49-58.

3. CARRILLO, A. 1998. Enfoques cualitativos y participativos en investigación social. Ed. UNAD (Bogotá). 245p. 
4. CANCELA, J.; AYÁN, C. 2011. Prevalencia y relación entre el nivel de actividad física y las actitudes alimenticias anómalas en estudiantes universitarias españolas de ciencias de la salud y la educación. Rev. Esp. Salud Pública. 85(5):499-505.

5. DODD, L.; AL-NAKEEB, Y.; NEVILL, A.; FORSHAW, M. 2010. Lifestyle risk factors of students: A cluster analytical approach. Preventive Medicine. 51:73-77. Disponible en Internet en: http://www.sciencedirect. com/science/article/pii/S0091743510001180 (con acceso 07/4/2014).

6. ESPINOZA, L.; RODRÍGUEZ, F.; GÁLVEZ, J.; MACMILLAN, N. 2011. Hábitos de alimentación y actividad física en estudiantes universitarios. Rev. Chilena de Nutrición. 38(4):458-465.

7. ESCOBAR, M.; PICO M. 2013. Autocuidado de la salud en jóvenes universitarios, Manizales, 2010-2011. Rev. Facultad Nacional de Salud Pública. (Colombia). 31(2):178-186.

8. FARINOLA, M.; BAZÁN, N. 2011. Conducta sedentaria y actividad física en estudiantes. Rev. Argentina Cardiología. 29(4):351-354.

9. GARCÍA, E.; MARTINI, J.; PORCEL, A. 2013. Alcohol consumption and risky sexual practices: the pattern of nursing students from the Spanish University. Rev. Latino-Am. Enfermagem, Ribeirão Preto. 21(4):941947.

10. GONZÁLEZ, C.; DÍAZ, Y.; MENDIZABAL, A.; MEDINA, E.; MORALES, J. 2014. Prevalencia de obesidad y perfil lipídico alterado en jóvenes universitarios. Nutrición Hospitalaria. (México). 29(2):315-321. Disponible desde Internet en: http://www.aulamedica.es/ gdcr/index.php/nh/article/viewFile/7054/pdf_52 (con acceso 20/2/2014).

11. GRIEKEN, A.; EZENDAM, N.; PAULIS, W.; VAN DER WOUDEN, J.; RAAT, H. 2012. Primary prevention of overweight in children and adolescents: a metaanalysis of the effectiveness of interventions aiming to decrease sedentary behavior. Int. J. Behav. Nutr. Phys. Act. (9):61-72. Disponible desde Internet en: http://www.ncbi.nlm.nih.gov/pmc/articles/ PMC3462110/ (con acceso 04/4/2014).

12. HAINES, R.; OLIFFE, J.; WHITE, C.; BOTTORFF, J. 2013. "It is just not part of the culture here": Young adults' photo-narratives about smoking, quitting, and healthy lifestyles in Vancouver, Canada. Health \& Place. 22(7):19-23. Disponible en Internet en: http://www.sciencedirect.com/science/article/pii/ S1353829213000312?np=y (con acceso 4/4/2014).

13. HERNÁNDEZ, O. 2006. Proyecto de vida y desarrollo integral humano. Rev. Internal Crecemos. (Puerto Rico). 6(1-20):1-31. Disponible desde Internet en: http:// biblioteca.clacso.edu.ar/ar/libros/cuba/cips/caudales05/Caudales/ARTICULOS/ArticulosPDF/07D050. pdf (con acceso 05/07/2012).

14. IGLESIAS, M.; MATA, G.; PÉREZ, A.; HERNÁNDEZ, S.; GARCÍA R.; PAPADAKI, C. 2013. Estudio nutricional en un grupo de estudiantes universitarios madrileños. Nutr. Clín. Diet Hosp. (España). 33(1):23-30.

15. JEONGSOO, KIM. 2014. Experiences of health related lifestyles in high body fat but non-obese female college students in korea. Osong Public Health Res. Perspect. 5(1):68-73. Disponible desde Internet en: http://www.sciencedirect.com/science/journal/22109099/5/1 (con acceso 04/4/2014).

16. LEAL, E. 2009. La Investigación Acción Participación, un aporte al conocimiento y a la transformación de Latinoamérica, en permanente movimiento. Rev. Invest. (Venezuela) 33(67):13-34.

17. MONTEIRO, C.; MANCUSSI, A. 2006. Actividad física según la percepción de estudiantes de enfermería. Rev. Latino-americana de Enfermagen. (Brasil). 14(6):17.

18. MOSQUERA, J.; ARTAMÓNOVA, I.; MOSQUERA, V. 2011 Consumo de sustancias psicoactivas en la Universidad Tecnológica de Pereira. Investigaciones Andina. (Colombia). 13(22):194-210.

19. PÁEZ CALA, M.L.; CASTAÑO CASTRILLÓN, J.J. 2010. Estilos de vida y salud en estudiantes de una facultad de Psicología. Psicología desde el Caribe 25:155178.

20. PALENCIA, E. 2006. Reflexión sobre el ejercicio docente de enfermería en nuestros días. Invest. Edu. Enfermería. (Colombia). 24(2):130-134.

21. PEDROSA, A.; CAMACHO, L.; PASSOS, S.; OLIVEIRA, R. 2011. Consumo de álcool entre estudantes universitários. Cad. Saúde Pública (Brasil). 27(8):16111621. 
22. REYES, D.; GALLEGO, G.; PÁEZ, S.; PULIDO, G. 2011. Fundamentación. Área temática de calidad de vida. Secretaría de Integración Social. (Bogotá-Colombia). 62p. Disponible en internet en http://www.integracionsocial.gov.co/anexos/documentos/Serviciosenlinea/Fundamentación\%20área\%20temática\%20 de\%20Calidad\%20de\%20Vida.pdf (con acceso 05/10/2012).

23. RIZO, N.; GONZÁLEZ, B.; CORTÉS, E. 2014. Calidad de la dieta y estilos de vida en estudiantes de Ciencias de la Salud. (España). Nutr Hosp. 29(1):153-15. Disponible desde Internet en: http://web.a.ebscohost. com.ezproxy.unal.edu.co/ehost/pdfviewer/ pdfviewer?vid $=3 \&$ sid $=56931388-$ dfb3-4e5e-ba510cd808bb8c61\%40sessionmgr4004\&hid $=4106$ (con acceso 06/04/014).

24. RODRÍGUEZ, F.; PALMA, X.; ROMO, A; ESCOBAR, D.; ARAGÚ, B.; ESPINOZA, L.; NORMAN, M.; GALVEZ, J. 2013. Hábitos alimentarios, actividad física, y nivel socioeconómico en estudiantes universitarios de Chile. Nutr Hosp. 28(2):447-455.

25. SCHNETTLER, B.; DENEGRI, M.; MIRANDA, H.; SEPÚLVEDA, J.; ORELLANA, L.; PAIVA, G.; GRUNERT, K. 2013. Hábitos Alimenticios y bienestar subjetivo en estudiantes universitarios del sur de chile. Nutr Hosp. 28(6):2221-2228. Disponible desde Internet en: http://www.nutricionhospitalaria.com/pdf/6751. pdf (con acceso 06/04/2014).
26. TERCEDOR, P.; MARTÍN, M.; PÉREZ, O.; WÄRNBERG, J.; DELGADO, M. 2007. Incremento del consumo de tabaco y disminución del nivel de práctica de actividad física en adolescentes españoles. Nutr. Hosp. 22(1):89-94.

27. VELÁSQUEZ, M.T.; TORRES-NEIRA, D.; SÁNCHEZMARTÍNEZ, H. 2006. Análisis psicológico de la actividad física en estudiantes de una universidad de Bogotá, Colombia. Salud Pública. (Colombia). 8(supl.2):1-12.

28. VIVES, A. 2007. Estilo de vida saludable: Puntos de vista para una opción actual y necesaria. Psicol. Cient. (La Habana, Cuba). 9(63):1-307. Disponible desde Internet en t:http://www.psicologiacientifica.com/ bv/psicologia-307-1-estilo-de-vida-saludable-puntos-de-vista-para-una-opcion-act.html (con acceso 26/09/2012).

29. ZÁRATE, M.; ZAVALETA, A.; DANJOY, D.; CHANAMÉ, E.; PROCHAZKA, R.; SALAS, M.; MALDONADO, V. 2006. Prácticas de consumo de tabaco y otras drogas en estudiantes de ciencias de la salud de una universidad privada de Lima, Perú. Rev. Investigación y Educación en Enfermería. 24(2):72-81.

Recibido: Agosto 13 de 2013

Aceptado: Abril 14 de 2014

\section{Como citar:}

Coral, R. del C.; Vargas, L.D. 2014. Estrategias que promueven estilos de vida saludables. Rev. U.D.C.A Act. \& Div. Cient. 17(1): 35-43. 
